\title{
Signal-Locality in Hidden-Variables Theories
}

\author{
Antony Valentini円 \\ Theoretical Physics Group, Blackett Laboratory, Imperial College, Prince \\ Consort Road, London SW7 2BZ, England.2 \\ Center for Gravitational Physics and Geometry, Department of Physics, The \\ Pennsylvania State University, University Park, PA 16802, USA. \\ Augustus College, 14 Augustus Road, London SW19 6LN, England. ${ }^{3}$
}

We prove that all deterministic hidden-variables theories, that reproduce quantum theory for a 'quantum equilibrium' distribution of hidden variables, predict the existence of instantaneous signals at the statistical level for hypothetical 'nonequilibrium ensembles'. This signal-locality theorem generalises yet another property of the pilot-wave theory of de Broglie and Bohm. The theorem supports the hypothesis that in the remote past the universe relaxed to a state of statistical equilibrium (at the hidden-variable level) in which nonlocality happens to be masked by quantum noise.

PACS: 03.65.Ud; 03.65.Ta; 03.67.-a

\footnotetext{
${ }^{1}$ email: a.valentini@ic.ac.uk

${ }^{2}$ Corresponding address.

${ }^{3}$ Permanent address.
} 


\section{Introduction}

Bell's theorem shows that any reasonable deterministic hidden-variables theory behind quantum mechanics has to be nonlocal [1]. If Specifically, for pairs of spin- $1 / 2$ particles in the singlet state, the outcomes of spin measurements at one wing must depend instantaneously on the axis of measurement at the other, distant wing. In this paper we show that there are instantaneous signals at the statistical level for hypothetical ensembles whose distribution differs from that of quantum theory. This generalises yet another property of de Broglie-Bohm pilot-wave theory to all deterministic hidden-variables theories.

Historically, Bell's theorem was inspired by the pilot-wave theory of de Broglie and Bohm [2-10]. Bell asked [11] if all hidden-variables theories have to be nonlocal like pilot-wave theory; he subsequently proved that they do [1]. A further property of pilot-wave theory - 'contextuality' - was proved to be universal (that is, was proved to be a property of all hidden-variables theories) by Kochen and Specker [12].5 It is natural to ask if there are any other features of pilot-wave theory that can be generalised. Here we prove that, indeed, pilotwave theory has yet another universal feature: the 'signal-locality theorem'.

In pilot-wave theory a system with wavefunction $\psi(x, t)$ has a definite configuration $x(t)$ at all times, with velocity $\dot{x}(t)=j(x, t) /|\psi(x, t)|^{2}$ where $j$ is the quantum probability current. To recover quantum theory one assumes that an ensemble of systems with wavefunction $\psi_{0}(x)$ begins with a distribution of configurations $\rho_{0}(x)=\left|\psi_{0}(x)\right|^{2}$ at $t=0$ (guaranteeing $\rho(x, t)=|\psi(x, t)|^{2}$ for all $t$ ). The Born probability distribution is assumed as an initial condition. In principle, however, the theory allows one to consider arbitrary initial distributions $\rho_{0}(x) \neq\left|\psi_{0}(x)\right|^{2}$ which violate quantum theory $[5,10,13,14]$. (The 'quantum equilibrium' distribution $\rho=|\psi|^{2}$ is analogous to thermal equilibrium in classical mechanics, and may be accounted for by an $H$-theorem $[13,15]$.) For two entangled particles at $A$ and $B$ with wavefunction $\psi\left(x_{A}, x_{B}, t\right)$, operations performed at $B$ (such as switching on an external potential) have an instantaneous effect on the motion of the individual particle at $A$. However, for a quantum equilibrium ensemble $\rho\left(x_{A}, x_{B}, t\right)=\left|\psi\left(x_{A}, x_{B}, t\right)\right|^{2}$, operations at $B$ have no statistical effect at $A$ : entanglement cannot be used for signalling at a distance. But this masking of nonlocality by statistical noise is peculiar to equilibrium: for an ensemble $\rho_{0}\left(x_{A}, x_{B}\right) \neq\left|\psi_{0}\left(x_{A}, x_{B}\right)\right|^{2}$ at $t=0$, changing the Hamiltonian at $B$ generally induces an instantaneous change in the marginal distribution at $A[16] .9$

This is the signal-locality theorem of pilot-wave theory: in general, there

\footnotetext{
${ }^{4}$ It is assumed that there is no common cause between the hidden variables and the measurement settings, and no backwards causation (so that the hidden variables are unaffected by the future outcomes). Bell's original paper addressed only the deterministic case. The later generalisations to stochastic theories are of no concern here.

${ }^{5}$ Even if the original paper erroneously claimed to prove the nonexistence of hidden variables. In pilot-wave theory, of course, quantum 'measurements' do not usually reveal the value of some pre-existing attribute of the system, and the Kochen-Specker theorem shows that all hidden-variables theories must share this feature.

${ }^{6}$ The signal may vanish for some special $\rho_{0} \neq\left|\psi_{0}\right|^{2}$, but not in general.
} 
are instantaneous signals at the statistical level if and only if the ensemble is in quantum nonequilibrium $\rho_{0} \neq\left|\psi_{0}\right|^{2}[16]$. We wish to show that the same is true in any deterministic hidden-variables theory.

\section{Bell Nonlocality}

Consider two spin-1/2 particles lying on the $y$-axis at $A$ and $B$ and separated by a large distance. For the singlet state $|\Psi\rangle=(|z+, z-\rangle-|z-, z+\rangle) / \sqrt{ } 2$, spin measurements along the $z$-axis at each wing always yield opposite results. But we are of course free to measure spin components along arbitrary axes at each wing. For simplicity we take the measurement axes to lie in the $x-z$ plane; their orientations may then be specified by the (positive or negative) angles $\theta_{A}$, $\theta_{B}$ made with the $z$-axis. In units of $\hbar / 2$, the possible values of outcomes of spin measurements along $\theta_{A}, \theta_{B}$ at $A, B$ - that is, the possible values of the quantum 'observables' $\hat{\sigma}_{A}, \hat{\sigma}_{B}$ at $A, B-$ are \pm 1 . Quantum theory predicts that for an ensemble of such pairs, the outcomes at $A$ and $B$ are correlated: $\left\langle\Psi\left|\hat{\sigma}_{A} \hat{\sigma}_{B}\right| \Psi\right\rangle=-\cos \left(\theta_{A}-\theta_{B}\right)$.

Let us now assume the existence of hidden variables $\lambda$ that determine the outcomes $\sigma_{A}, \sigma_{B}= \pm 1$ along $\theta_{A}, \theta_{B}$. And let us assume there exists a 'quantum equilibrium ensemble' of $\lambda$ - that is, a distribution $\rho_{e q}(\lambda)$ that reproduces the quantum statistics (where $\int d \lambda \rho_{e q}(\lambda)=1$ ). Each value of $\lambda$ determines a pair of outcomes $\sigma_{A}, \sigma_{B}$ (for given $\theta_{A}, \theta_{B}$ ); for an ensemble of similar experiments in which the values of $\lambda$ generally differ from one run to the next - one obtains a distribution of $\sigma_{A}, \sigma_{B}$, which is assumed to agree with quantum theory. In particular, the expectation value

$$
\overline{\sigma_{A} \sigma_{B}}=\int d \lambda \rho_{e q}(\lambda) \sigma_{A}\left(\theta_{A}, \theta_{B}, \lambda\right) \sigma_{B}\left(\theta_{A}, \theta_{B}, \lambda\right)
$$

must reproduce the quantum result $\left\langle\hat{\sigma}_{A} \hat{\sigma}_{B}\right\rangle=-\cos \left(\theta_{A}-\theta_{B}\right)$. Bell showed that this is possible only if one has nonlocal equations

$$
\sigma_{A}=\sigma_{A}\left(\theta_{A}, \theta_{B}, \lambda\right), \quad \sigma_{B}=\sigma_{B}\left(\theta_{A}, \theta_{B}, \lambda\right)
$$

in which the outcomes depend on the distant angular settings [1].7

In principle, the nonlocality might be just 'one-way', with only one of $\sigma_{A}$, $\sigma_{B}$ depending on the distant setting. For instance, one might have $\sigma_{A}=$ $\sigma_{A}\left(\theta_{A}, \theta_{B}, \lambda\right)$ but $\sigma_{B}=\sigma_{B}\left(\theta_{B}, \lambda\right)$, with nonlocality from $B$ to $A$ but not from $A$ to $B$.

\footnotetext{
${ }^{7}$ Here $\lambda$ are the initial values of the hidden variables, for example just after the source has produced the singlet pair. Their later values may be affected by changes in $\theta_{A}, \theta_{B}$, and writing $\sigma_{A}=\sigma_{A}\left(\theta_{A}, \theta_{B}, \lambda\right), \sigma_{B}=\sigma_{B}\left(\theta_{A}, \theta_{B}, \lambda\right)$ (where $\lambda$ are initial values) allows for this. See ref. [4], chapter 8 .
} 


\section{General Signal-Locality Theorem}

Now given a distribution $\rho_{e q}(\lambda)$, one can always contemplate - purely theoretically - a 'nonequilibrium' distribution $\rho(\lambda) \neq \rho_{e q}(\lambda)$, even if one cannot prepare such a distribution in practice. For example, given an ensemble of values of $\lambda$ with distribution $\rho_{e q}(\lambda)$, mathematically one could pick a subensemble such that $\rho(\lambda) \neq \rho_{e q}(\lambda)$.

The theorem to be proved is then the following: in general, there are instantaneous signals at the statistical level if and only if the ensemble is in quantum nonequilibrium $\rho(\lambda) \neq \rho_{e q}(\lambda)$.

Proof: Assume first that $\sigma_{A}$ has some dependence on the distant setting $\theta_{B}$. (Bell's theorem requires some nonlocal dependence in at least one direction.)

Now consider an ensemble of experiments with fixed settings $\theta_{A}, \theta_{B}$ and an equilibrium distribution $\rho_{e q}(\lambda)$ of hidden variables $\lambda$. In each experiment, a particular value of $\lambda$ determines an outcome $\sigma_{A}=\sigma_{A}\left(\theta_{A}, \theta_{B}, \lambda\right)$ at $A$. Some values of $\lambda$ yield $\sigma_{A}=+1$, some yield $\sigma_{A}=-1$. What happens if the setting $\theta_{B}$ at $B$ is changed to $\theta_{B}^{\prime}$ ?

The set $S=\{\lambda\}$ of possible values of $\lambda$ may be partitioned in two ways:

$$
S_{A+}=\left\{\lambda \mid \sigma_{A}\left(\theta_{A}, \theta_{B}, \lambda\right)=+1\right\}, \quad S_{A-}=\left\{\lambda \mid \sigma_{A}\left(\theta_{A}, \theta_{B}, \lambda\right)=-1\right\}
$$

where $S=S_{A+} \cup S_{A-}, S_{A+} \cap S_{A_{-}}=\emptyset$, and

$$
S_{A+}^{\prime}=\left\{\lambda \mid \sigma_{A}\left(\theta_{A}, \theta_{B}^{\prime}, \lambda\right)=+1\right\}, \quad S_{A-}^{\prime}=\left\{\lambda \mid \sigma_{A}\left(\theta_{A}, \theta_{B}^{\prime}, \lambda\right)=-1\right\}
$$

where $S=S_{A+}^{\prime} \cup S_{A-}^{\prime}, S_{A+}^{\prime} \cap S_{A-}^{\prime}=\emptyset$. (There could exist a pathological subset of $S$ that gives neither outcome $\sigma_{A}= \pm 1$, but this must have measure zero with respect to the equilibrium measure $\rho_{e q}(\lambda)$, and so may be ignored.) It cannot be the case that $S_{A+}=S_{A+}^{\prime}$ and $S_{A-}=S_{A-}^{\prime}$ for arbitrary $\theta_{B}^{\prime}$, for otherwise the outcomes at $A$ would not depend at all on the distant setting at $B$. Thus in general

$$
T_{A}(+,-) \equiv S_{A+} \cap S_{A-}^{\prime} \neq \emptyset, \quad T_{A}(-,+) \equiv S_{A-} \cap S_{A+}^{\prime} \neq \emptyset
$$

In other words: under a shift $\theta_{B} \rightarrow \theta_{B}^{\prime}$ in the setting at $B$, some values of $\lambda$ that would have yielded the outcome $\sigma_{A}=+1$ at $A$ now yield $\sigma_{A}=-1$; and some $\lambda$ that would have yielded $\sigma_{A}=-1$ now yield $\sigma_{A}=+1$.

Of the equilibrium ensemble with distribution $\rho_{e q}(\lambda)$, a fraction

$$
\nu_{A}^{e q}(+,-)=\int_{T_{A}(+,-)} d \lambda \rho_{e q}(\lambda)
$$

make the nonlocal 'transition' $\sigma_{A}=+1 \rightarrow \sigma_{A}=-1$ under the distant shift $\theta_{B} \rightarrow \theta_{B}^{\prime}$. Similarly, a fraction

$$
\nu_{A}^{e q}(-,+)=\int_{T_{A}(-,+)} d \lambda \rho_{e q}(\lambda)
$$

make the 'transition' $\sigma_{A}=-1 \rightarrow \sigma_{A}=+1$ under $\theta_{B} \rightarrow \theta_{B}^{\prime}$. 
Now with the initial setting $\theta_{A}, \theta_{B}$, quantum theory tells us that one half of the equilibrium ensemble of values of $\lambda$ yield $\sigma_{A}=+1$ and the other half yield $\sigma_{A}=-1$. (That is, the equilibrium measures of $S_{A+}$ and $S_{A-}$ are both $1 / 2$.) With the new setting $\theta_{A}, \theta_{B}^{\prime}$, quantum theory again tells us that one half yield $\sigma_{A}=+1$ and the other half yield $\sigma_{A}=-1$ (the equilibrium measures of $S_{A+}^{\prime}$ and $S_{A-}^{\prime}$ again being $\left.1 / 2\right)$. The $1: 1$ ratio of outcomes $\sigma_{A}= \pm 1$ is preserved under the shift $\theta_{B} \rightarrow \theta_{B}^{\prime}$, from which we deduce the condition of 'detailed balancing'

$$
\nu_{A}^{e q}(+,-)=\nu_{A}^{e q}(-,+)
$$

The fraction of the equilibrium ensemble that makes the transition $\sigma_{A}=+1 \rightarrow$ $\sigma_{A}=-1$ must equal the fraction that makes the reverse transition $\sigma_{A}=-1 \rightarrow$ $\sigma_{A}=+1$.

But for an arbitrary nonequilibrium ensemble with distribution $\rho(\lambda) \neq$ $\rho_{e q}(\lambda)$, the 'transition sets' $T_{A}(+,-)$ and $T_{A}(-,+)$ will generally have different measures

$$
\int_{T_{A}(+,-)} d \lambda \rho(\lambda) \neq \int_{T_{A}(-,+)} d \lambda \rho(\lambda)
$$

and the nonequilibrium transition fractions will generally be unequal,

$$
\nu_{A}(+,-) \neq \nu_{A}(-,+)
$$

The crucial point here is that $T_{A}(+,-)$ and $T_{A}(-,+)$ are fixed by the underlying deterministic theory, and are therefore independent of $\rho(\lambda)$.

Thus, if with the initial setting $\theta_{A}, \theta_{B}$ we would have obtained a certain nonequilibrium ratio of outcomes $\sigma_{A}= \pm 1$ at $A$, with the new setting $\theta_{A}, \theta_{B}^{\prime}$ we will in general obtain a different ratio at $A$. Under a shift $\theta_{B} \rightarrow \theta_{B}^{\prime}$, the number of systems that change from $\sigma_{A}=+1$ to $\sigma_{A}=-1$ is unequal to the number that change from $\sigma_{A}=-1$ to $\sigma_{A}=+1$, causing an imbalance that changes the outcome ratios at $A$. In other words, in general the statistical distribution of outcomes at $A$ is altered by the distant shift $\theta_{B} \rightarrow \theta_{B}^{\prime}$, and there is a statistical signal from $B$ to $A .8$

Similarly, if $\sigma_{B}$ depends on the distant setting $\theta_{A}$, one may define non-zero transition sets $T_{B}(+,-)$ and $T_{B}(-,+)$ 'from $A$ to $B$ '; and in nonequilibrium there will generally be statistical signals from $A$ to $B$.

In the special case of 'one-way' nonlocality, only one of the pairs $T_{A}(+,-)$, $T_{A}(-,+)$ or $T_{B}(+,-), T_{B}(-,+)$ has non-zero measure, and nonequilibrium signalling occurs in one direction only.

\section{Remarks}

The possibility of nonlocal signalling from $B$ to $A$ (or from $A$ to $B$ ) depends on the existence of finite transition sets $T_{A}(+,-), T_{A}(-,+)$ (or $T_{B}(+,-)$,

${ }^{8}$ The signal vanishes for special $\rho(\lambda) \neq \rho_{e q}(\lambda)$ that happen to have equal measures for the transition sets, but not in general. 
$\left.T_{B}(-,+)\right)$. The signal vanishes in equilibrium $\rho(\lambda)=\rho_{e q}(\lambda)$; while if $\rho(\lambda)$ is concentrated on just one of $T_{A}(+,-), T_{A}(-,+)$ (or on just one of $T_{B}(+,-)$, $\left.T_{B}(-,+)\right)$, then all the outcomes are changed by the distant shift. Thus the size of the signal - measured by the fraction of outcomes that change at a distance - can range from $0 \%$ to $100 \%$.

It is important to know the size of the transition sets, because if they have very tiny equilibrium measure, then to obtain an appreciable signal the nonequilibrium distribution $\rho(\lambda) \neq \rho_{e q}(\lambda)$ would have to be very far from equilibrium - that is, concentrated on a very tiny (with respect to the equilibrium measure) set. Bell's theorem guarantees that at least one of the pairs $T_{A}(+,-)$, $T_{A}(-,+)$ or $T_{B}(+,-), T_{B}(-,+)$ has non-zero equilibrium measure (for otherwise we would have a local theory); but it tells us nothing about the size of these sets: we know only that, by a detailed-balancing argument, $T_{A}(+,-)$ and $T_{A}(-,+)$ must have equal equilibrium measure, as must $T_{B}(+,-)$ and $T_{B}(-,+)$. In this sense, Bell's theorem tells us there must be some nonlocality hidden behind the equilibrium distribution, but not how much.

Given a specific hidden-variables theory, the transition sets may be determined and their equilibrium measures explicitly calculated. This has been done in detail for the case of pilot-wave theory [17]. It is found that the transition sets are generically rather large: for example, in a case where the measurement procedures are identical at the two wings - that is, use identical equipment and coupling - it is found that with initial settings $\theta_{A}=\theta_{B}=0$, under a shift $\theta_{B} \rightarrow \theta_{B}^{\prime}=\pi / 2$ at $B$ the equilibrium fraction of outcomes that change at $A$ is equal to $1 / 4$ (with $1 / 8$ changing from +1 to -1 and $1 / 8$ from -1 to +1 ) [17]. In this case, even a mild disequilibrium $\rho(\lambda) \neq \rho_{e q}(\lambda)$ entails a significant signal.

But is it possible to derive a general, theory-independent lower bound on the equilibrium measure of the transition sets?

The problem may be formulated more explicitly as follows. The quantity

$$
\alpha \equiv \nu_{A}^{e q}(+,-)+\nu_{A}^{e q}(-,+)
$$

(the sum of the equilibrium measures of $T_{A}(+,-)$ and $T_{A}(-,+)$ ) is the fraction of the equilibrium ensemble for which the outcomes at $A$ are changed under $\theta_{B} \rightarrow \theta_{B}^{\prime}$ (irrespective of whether they change from +1 to -1 or vice versa, the fractions doing each being $\alpha / 2$ ). There is a 'degree of nonlocality from $B$ to $A$ ', quantified by $\alpha=\alpha\left(\theta_{A}, \theta_{B}, \theta_{B}^{\prime}\right)$. Similarly, one may define a 'degree of nonlocality from $A$ to $B$ ', quantified by the fraction $\beta=\beta\left(\theta_{A}, \theta_{B}, \theta_{A}^{\prime}\right)$ of outcomes at $B$ that change in response to a shift $\theta_{A} \rightarrow \theta_{A}^{\prime}$ at $A$.

It is convenient to rewrite $\alpha$ and $\beta$ in a different form. The quantity $\frac{1}{2}\left|\sigma_{A}\left(\theta_{A}, \theta_{B}^{\prime}, \lambda\right)-\sigma_{A}\left(\theta_{A}, \theta_{B}, \lambda\right)\right|$ equals 1 if the outcome $\sigma_{A}$ changes under $\theta_{B} \rightarrow$ $\theta_{B}^{\prime}$, and vanishes otherwise. Since $\rho_{e q}(\lambda) d \lambda$ is by definition the fraction of the equilibrium ensemble for which $\lambda$ lies in the interval $(\lambda, \lambda+d \lambda)$, the fraction $\alpha$ for which $\sigma_{A}$ changes may be expressed as

$$
\alpha=\frac{1}{2} \int d \lambda \rho_{e q}(\lambda)\left|\sigma_{A}\left(\theta_{A}, \theta_{B}^{\prime}, \lambda\right)-\sigma_{A}\left(\theta_{A}, \theta_{B}, \lambda\right)\right|
$$


Similarly,

$$
\beta=\frac{1}{2} \int d \lambda \rho_{e q}(\lambda)\left|\sigma_{B}\left(\theta_{A}^{\prime}, \theta_{B}, \lambda\right)-\sigma_{B}\left(\theta_{A}, \theta_{B}, \lambda\right)\right|
$$

Now it is trivial to prove that the 'total degree of nonlocality' $\alpha\left(\theta_{A}, \theta_{B}, \theta_{B}^{\prime}\right)+$ $\beta\left(\theta_{A}, \theta_{B}, \theta_{A}^{\prime}\right)$ cannot vanish for all settings $\theta_{A}, \theta_{B}, \theta_{A}^{\prime}, \theta_{B}^{\prime}$. For $\alpha$ and $\beta$ are both non-negative; so if $\alpha+\beta=0$ then $\alpha=\beta=0$; and if this were true for all settings, $\sigma_{A}\left(\theta_{A}, \theta_{B}, \lambda\right)$ would have no dependence on $\theta_{B}$ and $\sigma_{B}\left(\theta_{A}, \theta_{B}, \lambda\right)$ would have no dependence on $\theta_{A}$ (apart from a possible set of $\lambda$ of equilibrium measure zero), in contradiction with Bell's theorem. But is it possible to derive some sort of lower bound on $\alpha+\beta$ ?

Positive lower bounds on $\alpha+\beta$, and on $\alpha$ or $\beta$ alone, have been derived assuming certain symmetries; and it has been checked in detail that these bounds are satisfied by pilot-wave theory [17]. But a general lower bound has not yet been obtained.

It should be possible to derive - without any extra assumptions - a general lower bound on the average total degree of nonlocality $\overline{\alpha+\beta}$, obtained by averaging $\alpha+\beta$ over all possible initial and final settings $\theta_{A}, \theta_{B}, \theta_{A}^{\prime}, \theta_{B}^{\prime}$. . Bell's theorem trivially implies that $\overline{\alpha+\beta}>0$, but something more is needed to obtain a positive lower bound.

Note that $\alpha$ and $\beta$ have a natural interpretation in terms of nonlocal information flow at the hidden-variable level. For an equilibrium ensemble of pairs, shifting the angle at $B$ alters a fraction $\alpha$ of the outcomes at $A$ (from +1 to -1 or vice versa). Thus $\alpha$ may be interpreted as the average number of bits of information per singlet pair transmitted nonlocally (in equilibrium) from $B$ to $A$, and similarly for $\beta$ from $A$ to $B$ [17]. Of course, in equilibrium this information flow is not visible at the statistical level, because as many outcomes flip from +1 to -1 as from -1 to +1 . But each individual change in outcome does represent, at the hidden-variable level, the transmission of one bit of 'subquantum information'.

Finally, we remark that since the signal-locality theorem generalises yet another property of pilot-wave theory (in addition to nonlocality and contextuality), one wonders if there remain still further properties of pilot-wave theory that are actually universal properties of hidden-variables theories generally.

\section{Conclusion and Hypothesis}

We have proved a general 'signal-locality theorem': in any deterministic hiddenvariables theory that reproduces quantum statistics for some 'equilibrium' distribution $\rho_{e q}(\lambda)$ of hidden variables $\lambda$, a generic 'nonequilibrium' distribution $\rho(\lambda) \neq \rho_{e q}(\lambda)$ would give rise to instantaneous signals at the statistical level (as occurs in pilot-wave theory).

Bell's theorem tells us that if hidden variables exist then so do instantaneous influences. But there is no consensus on what to conclude from this. Similarly,

\footnotetext{
${ }^{9}$ Alternatively, one might look at the maximum value $(\alpha+\beta)_{\max }$.
} 
one must distinguish between the signal-locality theorem proved above and what this author proposes to conclude from it.

It seems mysterious that nonlocality should be hidden by an all-pervading quantum noise. We have shown that any deviation from that noise would make nonlocality visible. It is as if there is a conspiracy in the laws of physics that prevents us from using nonlocality for signalling. But another way of looking at the matter is to suppose that our universe is in a state of statistical equilibrium at the hidden-variable level, a special state in which nonlocality happens to be hidden. The physics we see is not fundamental; it is merely a phenomenological description of an equilibrium state [16].

This view is arguably supported by quantum field theory in curved spacetime, where there is no clear distinction between quantum and thermal fluctuations [18]. On this basis it has been argued that quantum and thermal fluctuations are really the same thing [19]. This suggests that quantum theory is indeed just the theory of an equilibrium state, analogous to thermal equilibrium.

On this view it is natural to make the hypothesis that the universe began in a state of quantum nonequilibrium $\rho(\lambda) \neq \rho_{e q}(\lambda)$, where nonlocal signalling was possible, the relaxation $\rho(\lambda) \rightarrow \rho_{e q}(\lambda)$ taking place during the great violence of the big bang $[5,10,13,14,16]$. In effect, a hidden-variables analogue of the classical thermal heat death has actually occurred in our universe. (In the classical heat death, thermal energy cannot be used to do work; in the 'quantum heat death', nonlocality cannot be used for signalling.)

This hypothesis could have observable consequences. The nonlocal effects of disequilibrium may have played a role in homogenising the universe at early times [10]. In cosmological inflationary theories, early corrections to quantum fluctuations would change the spectrum of primordial density perturbations imprinted on the cosmic microwave background [10, 20]. And particles that decoupled at sufficiently early times could still be in quantum nonequilibrium today: thus, exotic particles left over from the very early universe might violate quantum mechanics $[10,13,14,20]$.

Acknowledgements. For helpful comments and discussions I am grateful to Guido Bacciagaluppi, Jossi Berkovitz, Lucien Hardy, Lee Smolin and Sebastiano Sonego, to audiences at the Universities of Maryland, Notre Dame and Utrecht, and to participants at the NATO workshop 'Modality, Probability, and

Bell's Theorems', Cracow, August 19-23, 2001. This work was supported by the Jesse Phillips Foundation.

\section{REFERENCES}

[1] J.S. Bell, Physics 1 (1965) 195.

[2] L. de Broglie, in: Electrons et photons (Gauthier-Villars, Paris, 1928). [English translation: G. Bacciagaluppi and A.Valentini, Electrons and photons: the proceedings of the fifth solvay congress (Cambridge University Press, forthcoming).] 
[3] D. Bohm, Phys. Rev. 85 (1952) 166, 180.

[4] J.S. Bell, Speakable and unspeakable in quantum mechanics (Cambridge University Press, 1987).

[5] A. Valentini, On the pilot-wave theory of classical, quantum and subquantum physics, PhD Thesis (1992), International School for Advanced Studies, Trieste.

[6] P. Holland, The quantum theory of motion (Cambridge University Press, 1993).

[7] D. Bohm and B.J. Hiley, The undivided universe (Routledge, 1993).

[8] J.T. Cushing, Quantum mechanics (Chicago, 1994).

[9] Bohmian mechanics and quantum theory, eds. J.T. Cushing et al. (Kluwer, 1996).

[10] A. Valentini, Pilot-wave theory of physics and cosmology (Cambridge University Press, forthcoming).

[11] J.S. Bell, Rev. Mod. Phys. 38 (1966) 447.

[12] S. Kochen and E. Specker, J. Math. Mech. 17 (1967) 59. 2001).

[13] A. Valentini, in: Chance in physics, eds. J. Bricmont et al. (Springer,

[14] A. Valentini, in ref. [9].

[15] A. Valentini, Phys. Lett. A 156 (1991) 5.

[16] A. Valentini, Phys. Lett. A 158 (1991) 1.

[17] A. Valentini, in: Modality, probability, and Bell's theorems, eds. T. Placek and J. Butterfield (Kluwer, 2002) quant-ph/0112151.

[18] D.W. Sciama, P. Candelas, and D. Deutsch, Adv. Phys. 30 (1981) 327.

[19] L. Smolin, Class. Quantum Grav. 3 (1986) 347.

[20] A. Valentini, Int. J. Mod. Phys. A, forthcoming. 